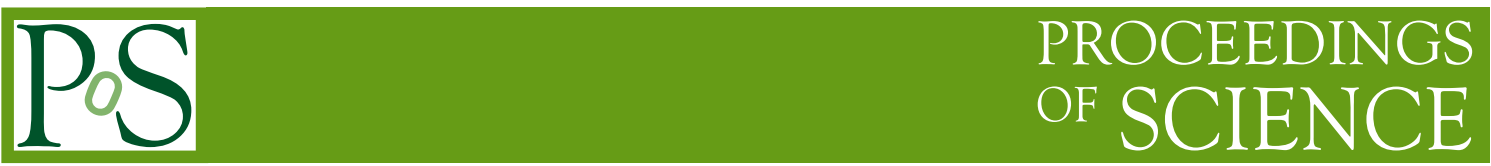

\title{
EBL and Cosmology Studies in the CTA Era
}

\section{Daniel Mazin*}

Institut de Fisica d'Altes Energies, Barcelona

E-mail: mazineifae.es

\begin{abstract}
Very high energy (VHE, E >100 GeV) gamma-rays are absorbed via interaction with low-energy photons from the extragalactic background light (EBL) if the involved photon energies are above the threshold for electron-positron pair creation. The VHE gamma-ray absorption, which is energy dependent and increases strongly with redshift, distorts the VHE energy spectra observed from distant objects. The observed energy spectra of the AGNs carry, therefore, an imprint of the EBL. The detection of hard VHE gamma-ray spectra of distant sources $(z=0.11-0.54)$ by H.E.S.S. and MAGIC enabled to set strong upper limits on the EBL density, using certain basic assumptions about blazar physics. These assumptions are, however, under discussion. In this paper, we give an overview of the EBL constraints, their limitations and perspectives for the joint efforts of the Fermi Gamma-Ray Space telescope, current imaging atmospheric Cherenkov telescopes and future project dubbed the Cherenkov Telescope Array (CTA)
\end{abstract}

AGN Physics in the CTA Era - AGN2011,

May 16-17, 2011

Toulouse, France

${ }^{*}$ Speaker. 


\section{Introduction}

During the star and galaxy formation history a diffuse extragalactic radiation field has been accumulated in the ultraviolet to far infrared wavelength regimes. This radiation field, commonly referred to as the extragalactic background light (EBL), is the second largest, in terms of the contained energy, background after the Cosmic Microwave Background of $2.7 \mathrm{~K}$ (CMB). While the $\mathrm{CMB}$ conserves the structure of the universe at the moment of the decoupling of matter and radiation following the Big Bang (at redshift $\mathrm{z} \approx 1000$ ), the EBL is a calorimetric measure of the entire radiant energy released by processes of structure formation that have occurred since the decoupling.

A closer look to the UV - infrared backgrounds is given in Figure 1, left plot. From right to left, the spectral energy distributions of the three major components are shown: the cosmic microwave background (CMB), the cosmic infrared background (CIB), and the cosmic optical background (COB). The COB, peaking at around $1 \mu \mathrm{m}$ is believed to originate directly from stars. The CIB, having its peak at $\sim 100 \mu \mathrm{m}$, results mostly from starlight that has been absorbed by dust inside galaxies and reemitted at higher wavelengths. Throughout this paper, we will refer to COB and CIB together as EBL. Other contributions, like emission from AGN and quasars are expected to produce no more than 5 to $20 \%$ of the total EBL density in the mid IR (see e.g. [1] and references therein).
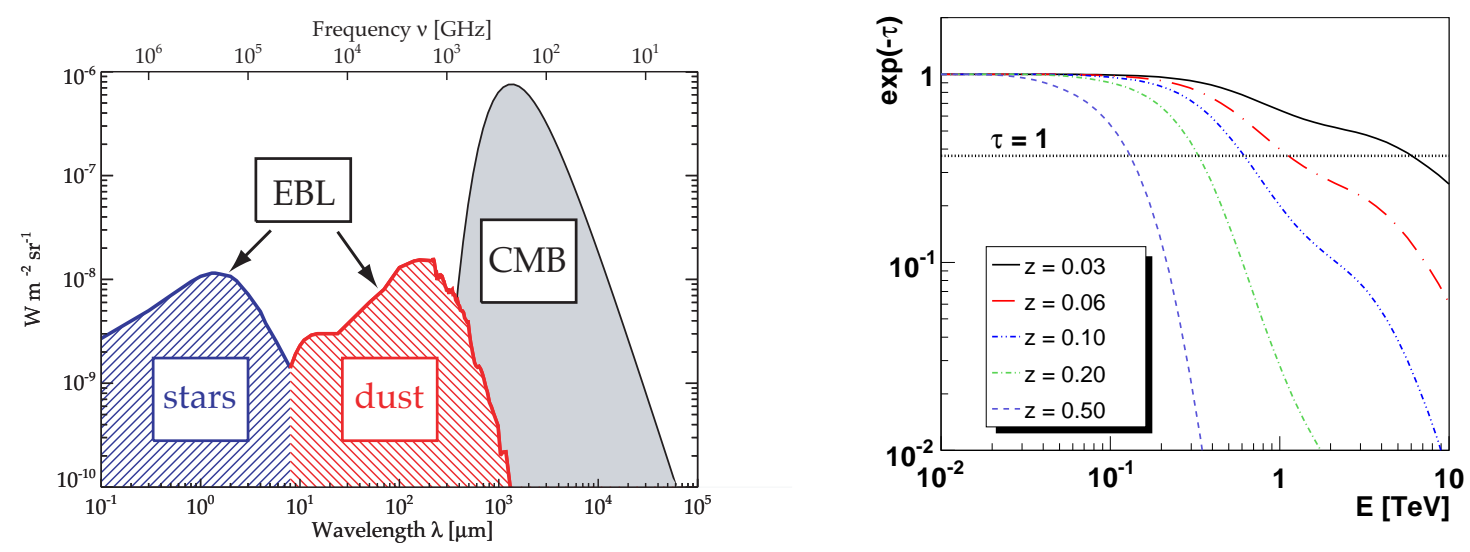

Figure 1: Left: Schematic Spectral Energy Distributions (SED) of the most important (by intensity) backgrounds in the universe. From right to left: the cosmic microwave background (CMB), the cosmic infrared background (CIB) and the cosmic optical background (COB). The last two components together are called EBL. Plot adopted from [⿰巾𠃌 Right: Attenuation coefficients $\exp (-\tau)$ as a function of $\gamma$-ray energy. The "best fit" model from [6] is used for the EBL density. The curves represent the expected attenuation of the VHE $\gamma$-ray spectra for different source distances. The horizontal dotted line corresponds to the optical depth $\tau=1$, i.e. it crosses the attenuation curves at the energy, above which the universe becomes opaque.

The EBL is difficult to measure directly due to strong foregrounds from our solar system and the Galaxy. The observation of distant sources of VHE $\gamma$-rays using Imaging Air Cherenkov Telescopes (IACT, such CANGAROO, H.E.S.S., MAGIC or VERITAS) provides a unique indirect measurement of the EBL (see below). The precision of the EBL constraints set by the IACT improved remarkably in the last few years. Contemporaneously with the IACT constraints, there 
has been rapid progress in resolving a significant fraction of this background with the deep galaxy counts at infrared wavelengths from the Infrared Space Observatory (ISO) and from the Spitzer satellite as well as at sub-millimeter wavelengths from the Submillimeter Common User Bolometer Array (SCUBA) instrument. The current status of direct and indirect EBL measurements (excluding limits from the IACTs) can be found in [3].

In total, the collective limits on the EBL between the UV and far-IR confirm the expected two peak structure, although the absolute level of the EBL density remains uncertain by a factor of 2 to 10. In addition to this consistent picture, several direct measurements in the near IR have also been reported (e.g. 㺻), significantly exceeding the expectations from source counts (see [ [7] and [8] for recent reviews). If this claimed excess of the EBL is real, it might be attributed to emissions by the first stars in the history of the universe. These so-called Population III stars are believed to be heavy stars with a very low metallicity.

This paper is structured as follows. In the next section we will describe the absorption effect on spectra of distant VHE $\gamma$-ray sources such as blazars due to the EBL and a possibility of using measured blazar spectra to constrain the EBL. Then we present the current status of the EBL constraints using blazar spectra and discuss their validity. Finally, we sketch possible improvements of the EBL measurements in the next five years using the new generation of the IACTs and the Fermi satellite.

\section{VHE $\gamma$-rays from blazars as a probe of the EBL}

On the way from the source to the observer, VHE $\gamma$-rays can suffer absorption losses by interaction with the low energy photons of the EBL. The corresponding reaction is the pair-production of an electron-positron pair:

$$
\gamma_{\mathrm{VHE}}+\gamma_{\mathrm{EBL}} \longrightarrow e^{+}+e^{-} \text {with } E_{\gamma_{\mathrm{VHE}}} \cdot E_{\gamma_{\mathrm{EBL}}}>\left(m_{e} c^{2}\right)^{2}
$$

The optical depth of the VHE $\gamma$-rays, $\tau(E)$, emitted at the redshift $z$, can then be calculated by solving the three-fold integral (see also [9]):

$$
\begin{aligned}
\tau\left(E_{\gamma}, z\right) & =\int_{0}^{z} \mathrm{~d} \ell\left(\mathrm{z}^{\prime}\right) \int_{-1}^{1} d \mu \frac{1-\mu}{2} \int_{\varepsilon_{t h}^{\prime}}^{\infty} d \varepsilon^{\prime} n\left(\varepsilon^{\prime}, z^{\prime}\right) \sigma_{\gamma \gamma}\left(\varepsilon^{\prime}, E^{\prime}, \mu\right) \\
\mu & :=\cos \theta \\
n(\varepsilon) & :=\text { EBL energy density } \\
\mathrm{d} \ell(\mathrm{z}) & :=\text { distance element }
\end{aligned}
$$

The expected optical depth for VHE $\gamma$-ray fluxes from sources at different redshifts is shown in Fig. 1, right panel. Hereby, the "best fit" model from [6] is used to estimate the EBL density. One can see that the optical depth is not only redshift but also energy dependent. The crossing points of the horizontal dotted line in Fig. 1 $1(\tau=1)$ with the attenuation lines define then the energy for the different redshifts, at which the universe becomes opaque. It is evident that a low threshold of a VHE $\gamma$-ray detector is essential to observe distant sources.

There are two major aspects concerning the interconnection between VHE $\gamma$-rays and the EBL: 
1. The EBL leaves a unique imprint on the VHE spectra. This imprint can be used to study the EBL.

2. The impact of EBL onto VHE spectra is fundamental. We need to understand EBL in order to study intrinsic properties of the VHE $\gamma$-ray sources.

Concerning point (1): From a single observed energy spectrum of a distant VHE $\gamma$-ray source, it is rather difficult if not impossible to distinguish between the imprint of the EBL and intrinsic features of the source. Observed features can be source inherent due to an internal absorption inside the source or due to a source, which does not provide necessary conditions for acceleration of charged particles to high enough energy. A way to pin-point the EBL impact is to use population studies of many extragalactic sources: whereas the intrinsic features might be different, the imprint of the EBL is the same (at a given redshift). With the current population of VHE $\gamma$-ray sources, it is only possible to set limits on the EBL, arguing that the observed spectra contain at least the imprint of the EBL.

Note that only distant extragalactic VHE $\gamma$-ray emitters suffer from the absorption by the EBL. For the galactic sources, the effect is negligible up to energies of about $100 \mathrm{TeV}$. For higher energies, the absorption by the photon field of the CMB starts to be important.

\section{Status of the EBL limits set by Cherenkov telescopes}

The observed VHE $\gamma$-ray spectrum of a source $\left(F_{\mathrm{obs}}\right)$ can be used to reconstruct the intrinsic spectrum $\left(F_{\text {intr }}\right)$, i.e. the one at the source location:

$$
F_{\text {intr }}=\exp (\tau) \times F_{\text {obs }},
$$

whereas $\tau$ is an energy and distance dependent optical depth of VHE $\gamma$-rays. By measuring the observed spectrum of a source and inferring certain limits on the intrinsic spectrum of the source, it is thus possible to constrain $\tau$ and, therefore, to constrain the EBL density.

Flat Spectrum Radio Quasars (FSRQ) and BL Lacs are subclasses of AGNs, which have their jet aligned close to the line of sight of the observer. The two subclasses are jointly called "blazars". According to an accepted paradigm, in blazars VHE $\gamma$-ray photons are produced by VHE electrons or protons, which are accelerated to VHE energies through a shock acceleration. High bulk motion Lorentz factors in the jets and the jet alignment towards the observer enhance chances to detect VHE $\gamma$-ray emission. Blazars are indeed the so far most prominent class of VHE $\gamma$-ray emitting extragalactic objects. In the shock acceleration models, the hardest index obtained for the accelerated particles is $s=1.5$ (see e.g. [10]). In the case of protons interacting with ambient plasma, the resulting $\gamma$-ray spectrum has the same slope as $s$, i.e. $\Gamma_{\text {int }}=1.5$. In the case of electrons, the spectrum of the $\gamma$-rays emitted through inverse Compton scattering is expected to be steeper than 1.5 under most circumstances. Therefore, different authors [11, 12, 13] assumed $\Gamma_{\text {int }}=1.5$ to be the hardest possible intrinsic spectrum, and using this criterion stringent EBL limits were derived. It was, however, argued that stochastic electron acceleration [14], truncated electron spectra [15] or internal absorption (e.g. [16, 17]) can lead to even harder VHE $\gamma$-ray spectra than with an index of $\Gamma_{\text {int }}=1.5$ although up to now no harder spectra with $\Gamma_{\text {int }}<1.5$ have been observed at lower energies, where no EBL absorption can take place. 

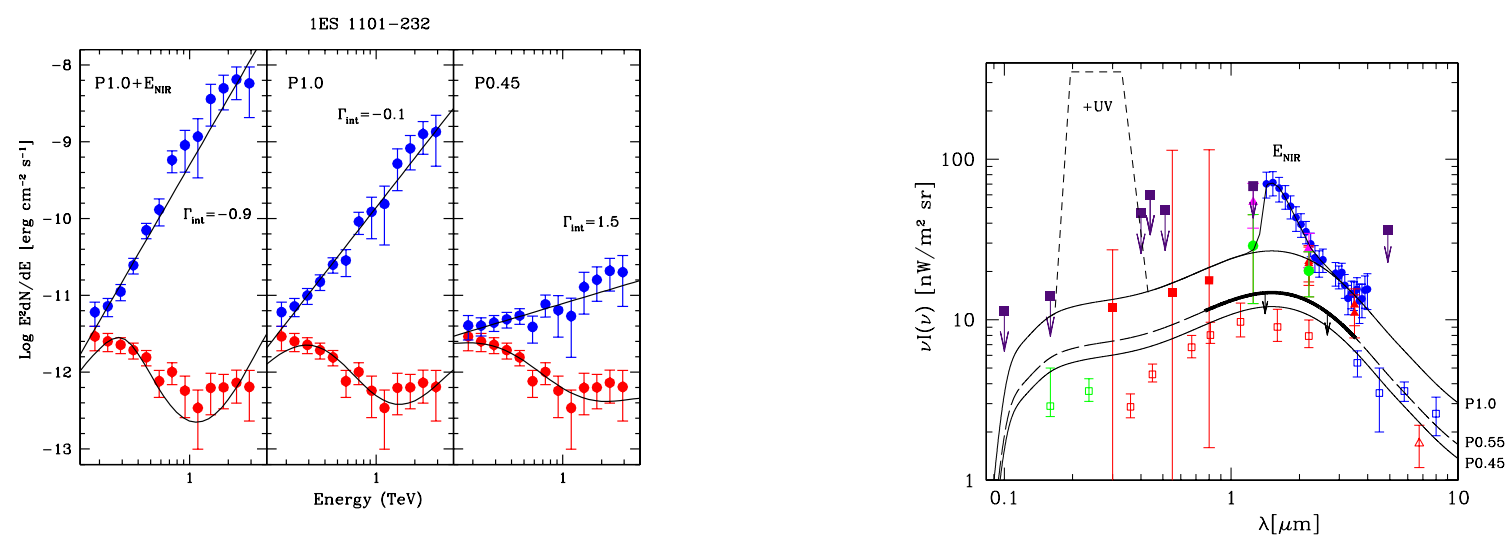

Figure 2: Left: The H.E.S.S. time-averaged spectrum of 1ES 1101-232 (red) together with a reconstructed intrinsic spectrum (blue) for three different EBL shapes. The first two EBL shapes lead to an unnaturally hard intrinsic spectrum (left and middle). Right: SED of the EBL. The thick black line between 0.8 and $4 \mu \mathrm{m}$ shows the H.E.S.S. limit. In the long-dashed regions, higher fluxes than the limit model would not be in conflict, as long as the flux in the $1-3 \mu \mathrm{m}$ range is within or around the limit. Figures from [12].

\subsection{H.E.S.S. limits on the EBL}

The H.E.S.S. collaboration published a detection of two intermediate redshift blazars 1ES 1101$232(\mathrm{z}=0.186)$ and H 2356-309 $(\mathrm{z}=0.165)$ [12]. Both observed spectra (measured in the range $150 \mathrm{GeV}-3 \mathrm{TeV}$ ) show a relatively hard spectral index of 2.9 and 3.1, respectively. Using the criterion that the intrinsic blazar spectrum cannot be harder than $\Gamma_{\text {int }}=1.5$, the authors derived a stringent upper limit on the EBL density in the region between 0.8 and $4 \mu \mathrm{m}$ (see Fig. 2). The derived upper limits imply a low level EBL density in agreement with the expectations from standard galaxy evolution models. The limits, in turn, rule out a cosmological origin of the near infrared

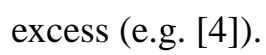

Using these EBL limits, physical parameters of Population III stars were explored by [18]. Sensitive limits on their star formation rate, metallicity and initial mass function were derived, which are comparable with or better than limits derived by other means (e.g. methods to derive the epoch of cosmic reionization).

\subsection{Combined EBL limits using all blazars until 2006}

A common criticism of the EBL limits derived as shown above is that they use only few blazars (therefore not providing consistency with other sources) and that the limits are obtained by assuming a certain EBL model and e.g. scaling it, or by exploring just a few details, i.e. the derived limits become very model-dependent. In order to avoid this dependency [5] performed a scan over many hypothetical EBL realizations (over 8 million different ones). The authors also tested all available blazar spectra (until 2006) to generalize the EBL limits. The scanned EBL 

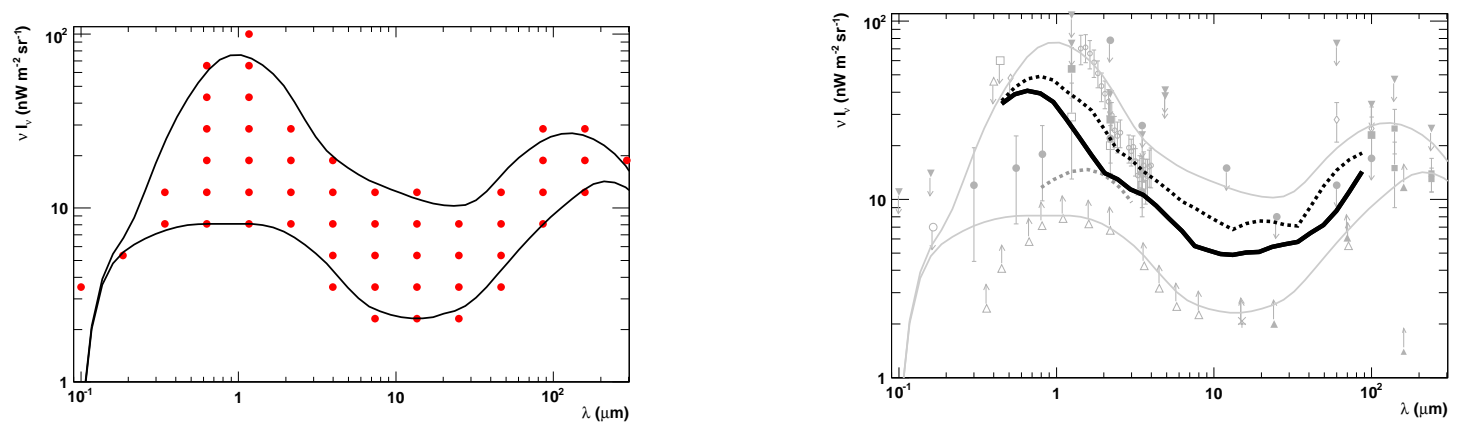

Figure 3: Combined results. Left: Grid points (red filled circles) and minimum and maximum shape of the scan. In total, 8064000 hypothetical EBL shapes were tested. Right: Combined results from the extreme scan (dashed black line) in comparison to the result from the realistic scan (solid black line). Grey lines are the minimum and the maximum shapes tested in the scan. Grey dotted line is the EBL upper limit from H.E.S.S. [?, aharonian:2006:hess:ebl:nature] Grey markers are direct measurements and limits. Figures from [5].

region is shown in Fig. 3, left plot. The derived upper limits on the EBL density are shown in the right plot of Fig. 3. Two limits are shown: the solid line represents the upper limit assuming that the intrinsic blazar spectrum cannot be harder than $\Gamma_{\mathrm{int}}=1.5$, whereas the dashed line shows the limit for $\Gamma_{\mathrm{int}}=2 / 3$. The latter one can be understood as the most conservative one as it is derived for monoenergetic electrons, which are responsible for the inverse Compton scattering of ambient photons. One can see that the derived limits favor a low EBL level and are in good agreement with galaxy counts from the optical to the mid infrared regimes. Again, the cosmological origin of the near infrared excess (e.g. [4]) can be ruled out even for the extreme case of $\Gamma_{\text {int }}=2 / 3$.

\subsection{MAGIC limits on the EBL}
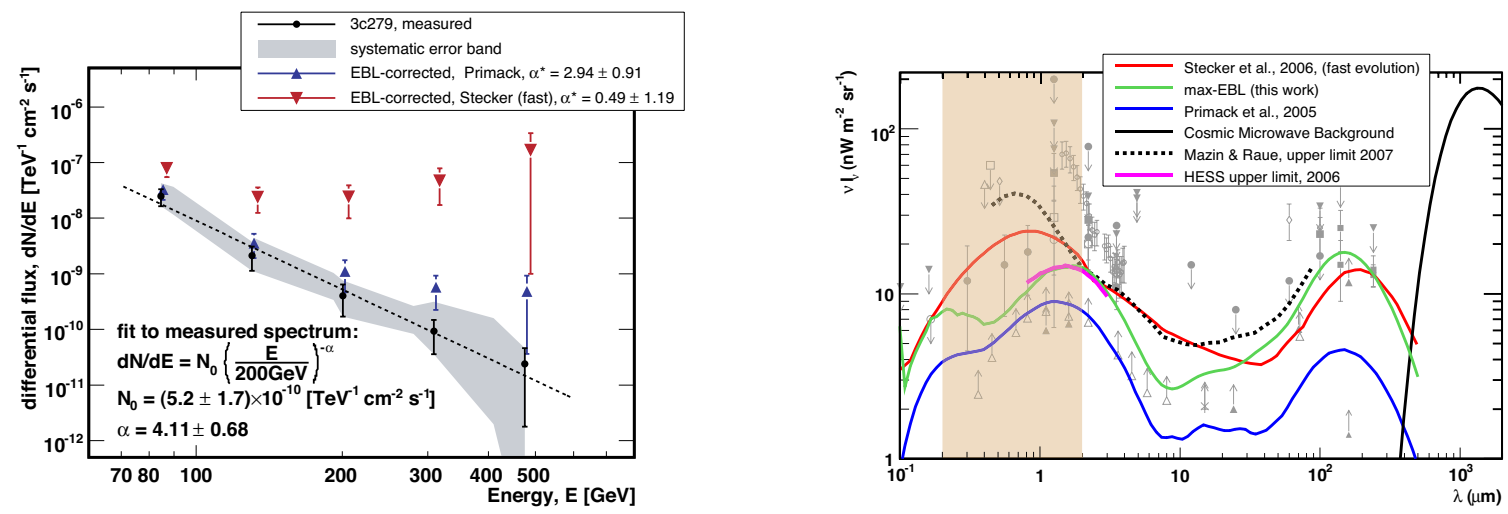

Figure 4: MAGIC Left: Spectrum of 3C 279 measured by MAGIC. The grey area includes the combined statistical $(1 \sigma)$ and systematic errors. The dotted line shows the result of a power law fit. The blue and red triangles are measurements corrected on the basis of two models for the EBL density: [19] (blue) and [20] (red). Right: SED of the EBL. Some of the EBL models are shown: [19] (blue), [20] (red) and [13] (green). The latter one corresponds to the derived EBL limit. The shaded vertical band indicates the range of frequencies where the MAGIC measurement is most sensitive. 
In 2007, the MAGIC collaboration reported a detection of a very distant $(\mathrm{z}=0.536)$ radio quasar 3C 279 at energies above $80 \mathrm{GeV}$ [21, 13]. The measured energy spectrum of 3C 279 extends up to $\approx 500 \mathrm{GeV}$ (see Fig. 4, left plot), which implies a very low EBL level. In order to derive an EBL limit, the MAGIC collaboration used a realistic EBL model of [6]. The authors [13] fine-tuned physical parameters of the EBL model in order to comply with the requirement that the intrinsic spectrum of $3 \mathrm{C} 279$ cannot be harder than $\Gamma_{\text {int }}=1.5$. The resulting maximum allowed EBL model is shown by the green line in Fig. 4, right plot. The EBL limit derived in [13] not only confirms limits from [12] and [5] but also probes for the first time the EBL at higher redshifts $0.2<$ $\mathrm{z}<0.5$. Moreover, due to a low energy threshold of MAGIC, the limit extends into the ultraviolet regime: the EBL region between 0.2 and $0.8 \mu \mathrm{m}$ has been probed for the first time. ${ }^{1}$

\subsection{Discussion of the limits}

Commenting the MAGIC result [13] some doubts arose if the same criterion of $\Gamma_{\text {int }}=1.5 \mathrm{can}$ be applied to 3C 279 (e.g. [25, 24]). A possible problem is that 3C 279 is not a blazar since (in a low flux state) it has prominent optical lines, identifying low energy (optical - infrared) radiation fields, usually called "broad line regions", BLR. Indeed, the presence of BLR is required in most leptonic models describing the broad band emission (from radio through VHE $\gamma$-rays) of 3C 279. The BLR in front or within the emission region of VHE $\gamma$-rays leads to an internal absorption of these $\gamma$-rays, which modifies the intrinsic spectrum. For some specific combinations of the geometry between the emission regions and the BLR combined with an assumption of a narrow band spectrum of the BLR (e.g., a black body spectrum), local hardening of the intrinsic VHE $\gamma$-ray spectrum can be expected (e.g. [17]). These question were addressed by [23] in detail. The authors find that, assuming a detailed BLR emission spectrum, for all plausible geometry combinations no significant hardening of the intrinsic spectrum of 3C 279 can be expected within the energy range observed by MAGIC. The authors also examined the EBL limits including the effect of the internal absorption finding that identical or even more stringent limits can be derived as compared to the ones obtained in [13].

Summarizing the status of the EBL constraints obtained by the IACTs, the following can be stated:

- robust EBL upper limits are derived by different groups extending from ultraviolet through mid infrared regimes;

- the limits are close (at most factor of 2 higher) to the EBL low level inferred from the resolved galaxies by HST, ISOCAM, Spitzer and the ground based telescopes (Keck, Subaru, VLT);

- the galaxy count slopes in the faint end in the optical and near-infrared bands also indicate that the majority of EBL has already been resolved (e.g. [26]);

- this implies that the above mentioned instruments resolved most of the EBL sources;

- the resulting $\gamma$-ray horizon can be determined to lie within a narrow band between the upper limits from the IACTs and the low limits from the galaxy counts (see blue shaded band in Fig. 5);

\footnotetext{
${ }^{1}$ Stecker \& Scully [22] argued, however, that the derived limit has a low significance.
} 


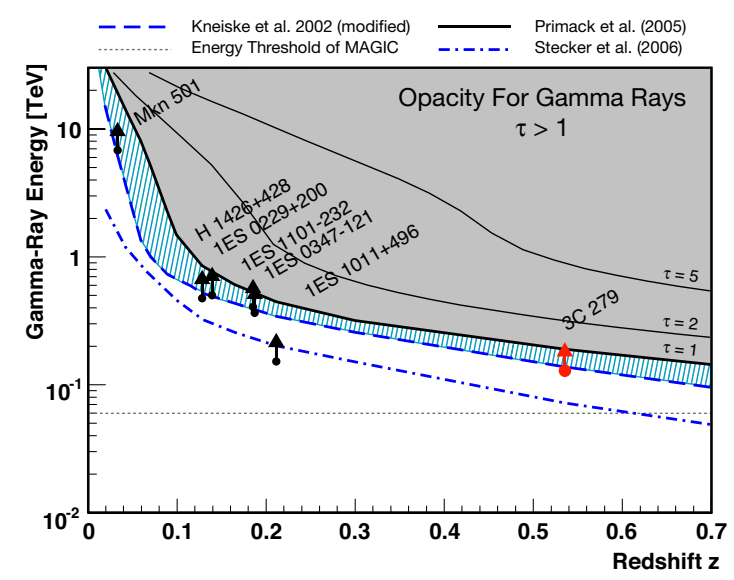

Figure 5: Gamma-ray horizon. IACTs probed the EBL density up to $z=0.536$. The derived limits leave only a narrow region (indicated by a blue band) for EBL models. Figure from [13].

- the limits disfavor several EBL models which imply a late peak in the star formation history;

- the limits rule out a cosmological origin of the near infrared excess.

However, the utilized exclusion criterion of the blazar intrinsic spectral index (being $\Gamma_{\text {int }}=$ 1.5 or even 2/3) can still be wrong. Even for an EBL model tuned to the level of the resolved galaxies [27], the intrinsic spectra of several $\mathrm{TeV}$ blazars show the maximum realistic hardness of 1.5. This can be related to the selection effect: only blazars with extremely hard spectra can be detected because the flux of blazars with softer spectra falls below the current sensitivity limit of the IACTs. Harder than expected intrinsic spectra of VHE $\gamma$-ray sources would imply either an unnatural fine-tuning of low energy radiation fields inside the sources, different acceleration mechanisms of charged particles responsible for VHE $\gamma$-ray emission or even new physics (e.g. violation of Lorentz invariance [28] or new particles [29]). However, the first results obtained by the Fermi/LAT team [30] indicate that the spectra of BL Lacs and FSRQs below $100 \mathrm{GeV}$ can be well described by power laws or broken power laws with spectral indeces $\Gamma_{\text {int }}>1.4$. This underlines robustness of the EBL limits obtained using the IACT data.

It is interesting to see that the current state-of-the-art EBL models [27, 31, 32, 33] (all published in the last 2 years) predict low EBL densities and show only a little spread among each other. Though the EBL models are derived following different approaches, they all seem to agree that most of the sources in the universe which contributed to the EBL are already resolved.

\section{Future perspective: Fermi/LAT, MAGIC II, H.E.S.S. II, VERITAS and CTA}

Currently, about 35 extragalactic VHE $\gamma$-ray emitters have been detected with redshifts up to $\mathrm{z}=0.536$. Although some of the measured spectra have very good statistics, there is a general difficulty to distinguish between source intrinsic effects (e.g. cut-off corresponding to the highest energies of relativistic particles in the jet or internal absorption due to local low energy photon fields) and the attenuation imprint caused by the EBL. The discovery of extragalactic sources with hard energy spectra by the HEGRA, H.E.S.S. and MAGIC instruments allowed to set stringent 
limits on the EBL density in the near to mid infrared (NIR and MIR) regimes [12, 5]. The limits are, however, dependent on the underlying sources physics assumptions, which are still a matter of debate. We anticipate that MAGIC II, H.E.S.S. II and VERITAS will at least double the statistics of the sources but this will most likely not resolve the ambiguity between intrinsic effects and the EBL. Additional caveat for the EBL limits from IACTs are rather high observational systematics in measuring VHE spectra. This is difficult to test in a direct way, compared with the direct gammaray detection instrument like Fermi/LAT. Future correlation study of Fermi/LAT and CTA spectra (see below) may give an answer.

The Fermi/LAT telescope measuring $\gamma$-rays between $100 \mathrm{MeV}$ and $300 \mathrm{GeV}$ from space is currently providing the best synergy with the IACTs by extending the energy spectra of extragalactic sources to lower energies. At energies below $50 \mathrm{GeV}$ there is basically no absorption of $\gamma$-ray fluxes due to the EBL. The Fermi/LAT, therefore, directly measures intrinsic spectra of the sources. First studies combining Fermi/LAT and IACT data [34, 35] strengthen the existing EBL limits in UV to mid IR. The studies, however, suffer from fast variability of the sources at very high energies, which cannot be measured by Fermi/LAT due to the relatively small $\left(0.8 \mathrm{~m}^{2}\right)$ collection area of the telescope and of the still small number of sources which are bright from few $\mathrm{GeV}$ to $1 \mathrm{TeV}$. Such sources are necessary to get enough photon statistics in the same time with the Fermi/LAT and with IACTs.

Probably, only $\mathrm{CTA}^{2}$ will be able to provide a sufficiently large sample (100++) of VHE $\gamma$ ray sources as well as high quality spectra for individual objects. For many of these sources, the spectral energy distribution (SED) will be determined at GeV energies (combining Fermi/LAT and lowest energies of the CTA measurements), which are much less affected by the absorption and, therefore, allowing for a study of intrinsic properties of the objects. We, therefore, anticipate that with CTA it will be possible to make robust predictions about the intrinsic spectrum above $100 \mathrm{GeV}$, either for individual sources or particular source classes. The measured spectra will therefore be a unique probe of the EBL. In the next 5 to 10 years following two main cosmological questions can be targeted: 1) precision measurements of today's (i.e. at $\mathrm{z}=0$ ) EBL, especially in the ultraviolet to optical as well as in the mid to far infrared regimes, and 2) determine galaxy and stellar evolution properties by measuring the evolution of the EBL over redshift. A first detailed study on the CTA potential in studyng the EBL can be found in [36].

\section{Acknowledgments}

D.M. thanks the MAGIC collaboration and in particular MAGIC IFAE group in Barcelona for the excellent support. D.M. also thanks Martin Raue for many fruitful discussions.

\section{References}

[1] I. Matute, F. La Franca, F. Pozzi, et al., Astronomy \& Astrophysics 451, 443-456 (2006).

[2] H. Dole, G. Lagache, Puget, et al., Astronomy \& Astrophysics 451, 417-429 (2006).

\footnotetext{
${ }^{2}$ planned next generation IACT array with a milli Crab sensitivity from $30 \mathrm{GeV}$ through several $100 \mathrm{TeV}$, arXiv:1008.3703
} 
[3] M. Raue \& D. Mazin Proceedings of SciNeGHE, 2010, arXiv:1106.4384.

[4] T. Matsumoto, S. Matsuura, H. Murakami, M. Tanaka, M. Freund, M. Lim, M. Cohen, M. Kawada \& M. Noda, The Astrophysical Journal 626, 31-43 (2005), astro-ph/ 0411593.

[5] D. Mazin \& M. Raue, Astronomy \& Astrophysics 471, 439-452 (2007), arXiv:astro-ph/0701694.

[6] T. M. Kneiske, K. Mannheim \& D. H. Hartmann, Astronomy \& Astrophysics 386, 1 (2002).

[7] M. G. Hauser \& E. Dwek, Annual Review of Astronomy \& Astrophysics 39, 249 (2001).

[8] A. Kashlinsky, Phys. Rep. 409, 361-438 (2005), astro-ph / 0412235.

[9] E. Dwek \& F. Krennrich, The Astrophysical Journal 618, 657-674 (2005), ast ro-ph / 0406565.

[10] M. A. Malkov \& L. O’C Drury, Reports on Progress in Physics 64, 429-481 (2001).

[11] F. A. Aharonian, A. G. Akhperjanian, M. Beilicke, et al., Astronomy \& Astrophysics 403, 523 (2003), astro-ph/0301437.

[12] F. Aharonian, A. G. Akhperjanian, A. R. Bazer-Bachi, et al., Nature 440, 1018-1021 (2006).

[13] J. Albert, E. Aliu, H. Anderhub, et al., Science 320, 1752- (2008), arXiv: 0807.2822.

[14] F. W. Stecker, M. G. Baring \& E. J. Summerlin, The Astrophysical Journal, Letters 667, L29-L32 (2007), arXiv:0707.4676.

[15] K. Katarzyński, G. Ghisellini, F. Tavecchio, J. Gracia \& L. Maraschi, MNRAS 368, L52-L56 (2006), arXiv:0603030.

[16] J. Sitarek \& W. Bednarek, Ap\&SS 309, 105-109 (2007), arXiv : 0610313.

[17] F. A. Aharonian, D. Khangulyan \& L. Costamante, MNRAS 387, 1206-1214 (2008).

[18] M. Raue, T. Kneiske \& D. Mazin, Astronomy \& Astrophysics 498, 25 (2009) arXiv: 0806.2574.

[19] J. R. Primack, J. S. Bullock \& R. S. Somerville, Observational Gamma-ray Cosmology, in High Energy Gamma-Ray Astronomy, edited by F. A. Aharonian, H. J. Völk \& D. Horns, 2005, vol. 745 of American Institute of Physics Conference Series, pp. 23-33.

[20] F. W. Stecker, M. A. Malkan, \& S. T. Scully, The Astrophysical Journal 648, 774-783 (2006), arXiv:astro-ph/0510449.

[21] M. Teshima et al., Proc. 30th Int. Cosm. Ray Conf. 3, 1045-1048 (2008), arXiv: 0709.1475.

[22] F. Stecker \& S. Scully, The Astrophysical Journal, Letters 691, p. L91 (2009).

[23] F. Tavecchio \& D. Mazin, MNRAS 392, L40 (2009), arXiv : 0809.2467.

[24] Costamante, L., Aharonian, F., Bühler, R., Khangulyan, D., Reimer, A., Reimer, O., AIPC 1085, 644-647 (2008), arXiv:0907.3966.

[25] J. Sitarek \& W. Bednarek, MNRAS 391, 624-638 (2008), arXiv: 0807 . 4228.

[26] T. Totani, Y. Yoshii, F. Iwamuro, T. Maihara \& K. Motohara, The Astrophysical Journal, Letters 550, L137-L141 (2001).

[27] A. Franceschini, G. Rodighiero \& M. Vaccari, Astronomy \& Astrophysics 487, 837-852 (2008), arXiv:0805.1841.

[28] R. J. Protheroe \& H. Meyer, Physics Letters B 493, 1 (2000). 
[29] A. de Angelis, M. Roncadelli \& O. Mansutti, Phys. Rev. D 76, 121301 (2007), arXiv: 0707.4312.

[30] A. A. Abdo et al., ApJS 187, 460-494 (2010), arXiv: 0910.1608.

[31] R. Gilmore et al. MNRAS 399, 1694 (2009)

[32] A. Dominguez et al., MNRAS in press (2010), arxiv: 1007.1459

[33] T. M. Kneiske \& H. Dole, Astronomy \& Astrophysics 515, 19 (2010)

[34] N. Mankuzhiyil, M. Persic \& F. Tavecchio, The Astrophysical Journal 715, L16-L20 (2010), arXiv:1004.2032

[35] M. Georganopoulos, J. D. Finke \& L. C. Reyes, The Astrophysical Journal 714, L157-L161 (2010), arXiv: 1004.0017

[36] M. Raue \& D. Mazin, Astropart. Phys. 34, 245-256 (2010), arXiv: 1005.1196 